\title{
Optimization of transmission and focusing properties of plasmonic nanolenses
}

\author{
P. Wróbel ${ }^{* a}$, T.J. Antosiewicz ${ }^{\mathrm{b}, \mathrm{c}}$, T. Szoplik ${ }^{\mathrm{a}}$ \\ ${ }^{a}$ University of Warsaw, Faculty of Physics, Pasteura 7, 02-093 Warsaw, Poland; \\ bInterdisciplinary Centre for Mathematical and Computational Modelling, University of Warsaw, \\ Pawińskiego 5A, 02-106 Warsaw, Poland \\ cChalmers University of Technology, Condensed Matter Theory, SE-412 96 Göteborg, Sweden.
}

\begin{abstract}
We consider two kinds of plasmonic nanolenses which focus radially polarized Laguerre-Gauss beam into subwavelength spot. The first one is free-standing opaque metal layer with concentric grooves on both sides [Phys. Rev. Lett. 102, 183902 (2009)]. The second has slits instead of grooves thus concentric rings have to be integrated with dielectric matrix. Constructive interference of far-field radiation of SPPs scattered on the back side of the lenses gives subwavelength size foci approaching the Rayleigh resolution limit. We investigate transmission and focusing properties of considered metal structures. Choice of appropriate metal such as silver, gold, copper or aluminum strongly affects transmission. Parameters of surface structure determine efficient photon-plasmon coupling and plasmon scattering phenomenon thus influence both transmission and focusing effect. Finally, the choice of dielectric function of surrounding medium gives another degree of freedom to fulfill momentum matching condition for resonant photon-plasmon interaction. In this paper, taking into account the above parameters, we show an optimization procedure, which leads to high transmission, tight focal spot and large focal length of the considered plasmonic nanolenses.
\end{abstract}

Keywords: plasmonics, plasmonic lens, radial polarization

\section{INTRODUCTION}

Possibility of squeezing light into sub-wavelength regions thank to conversion of photons to surface plasmon-polaritons (SPP) opened up a new way for miniaturization of photonic devices [1]. Through the last decade a lot of work was devoted to plasmonic focusing devices [2] which are in the form of planar metallic layers with holes, slits or surface corrugations either linear or circular [3-11]. Such structures, unlike classical, refractive lenses, concentrate light in a two-step process of photon-plasmon and plasmon-photon couplings. Such lenses can be classified as near-field devices concentrating evanescent field in the vicinity of the metal plate due to constructive interference of plasmons efficiently generated on circular grating [4-5], or far-field ones focusing light in the region distant a few wavelength from the lens due to phase matching of propagating beams scattered on the edges of slits or grooves at the back surface [6-12]. Generation an electromagnetic surface waves is strongly polarization dependent. Metal lenses with linear slits efficiently guide energy for p-polarized light and produce non-symmetric focal spot. For lens with concentric grooves or slits efficient photon-toplasmon coupling is possible when structure is illuminated by radially polarized beam. Then well shaped, symmetrical focal spot is formed. In this paper, we investigate two kinds of plasmonic nanolenses. The first has a form of either freestanding or integrated with dielectric substrate single metal layer with concentric grooves on both sides and no hole on the axis [13]. The second nanolens is composed of concentric slits integrated with dielectric matrix. Our earlier investigations were devoted to analysis of influence of geometric and material parameters on focusing and transmission properties of both structures [14]. Here we analyze integration of both lenses with dielectric matrix and influence of refractive index of a dielectric on optical properties of considered structures. The analysis is performed for the spectral range $450 \div 700 \mathrm{~nm}$ and similar values of groove/slit lattice.

* piotr.wrobel@igf.fuw.edu.pl; phone +48 0225546896 


\section{METAL NANOLENSES WITH GROOVES AND SLITS}

\subsection{Characteristics of nanolenses}

Figure 1 (left) shows a single silver layer nanolens with grooves on both sides and no hole on the axis and another one (right) made of concentric slits. Both structures are integrated with dielectric substrate and thin, anti-corrosion superstrate. Grooves and slits are filled with the same dielectric of refractive index $n$ as core and cladding of a fiber which guides illuminating light. Structures are described by the following parameters: metal layer thickness $d$, slit and groove width $w$, lattice constant $\Lambda$, groove depth $h$ and on-axis stop diameter s. Diameter of both nanolenses is $2 r$.In simulations the following parameters are kept constant: $\mathrm{d}=100 \mathrm{~nm}, \mathrm{w}=100 \mathrm{~nm}$ and $\mathrm{h}=40 \mathrm{~nm}$. To achieve efficient photon-to-plasmon coupling both structures are illuminated by radially polarized Laguerre-Gaussian doughnut mode [15] of maximum beam intensity $\mathrm{R}=1.7 \mu \mathrm{m}$.

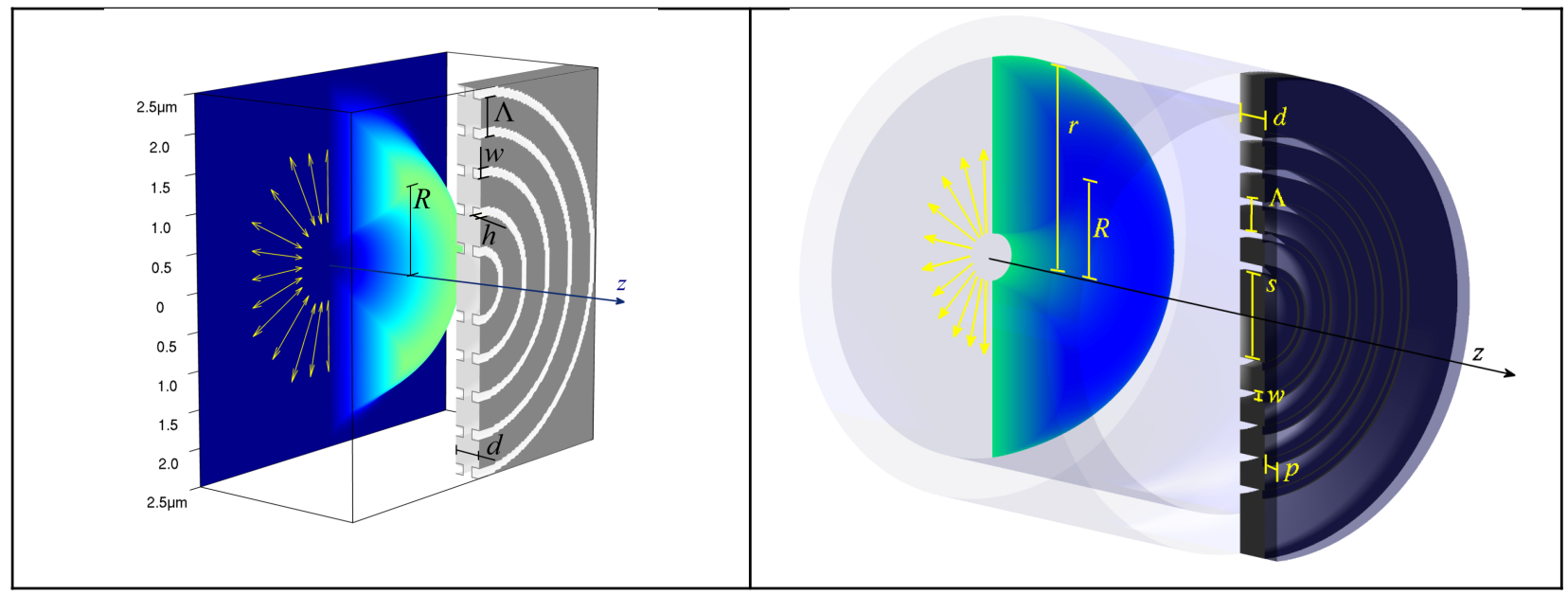

Fig. 1. (Left) Free standing nanolens with five concentric grooves with lattice constant $\Lambda$. (Right) Nanolens with five slits embeded into dielectric matrix.

Focus formation by both lenses is a two-step process of photon-to-plasmon and plasmon-to-photon conversion. In the first step incident photons efficiently couple to SPPs on the front surface due to periodic grating of grooves or slits which give extra spatial frequency defined as reciprocal vector of the grating $\mathrm{k}_{\mathrm{g}}=2 \pi / \Lambda$. The wavevector of the grating allows for momentum matching of impinging light and SPP wave described by the following equation,

$$
k_{S P P}=k_{0} n \sin \varphi+q k_{g} \text {, }
$$

where $k_{0}$ is the wavevector in free space, $\varphi$ is the angle of incidence, $n$ is the refractive index of a dielectric matrix, and $q$ is an integer.

Due to radially polarized illumination with Laguerre-Gauss beam with intensity profile of axial symmetry [16-18] plasmons are uniformly excited on the whole perimeters of front side grooves or slits. The optical axis of incident beam with radial beam profile of electric field

$$
E_{r}(r)=\left(\frac{r}{R}\right) \exp \left(-\frac{r^{2}}{2 R^{2}}\right),
$$

where $\mathrm{R}$ is a radius of maximum intensity, coincides with the axis of symmetry of grooves and slits. Proper choice of beam diameter adjusted to radius of corrugated or perforated areas maximizes efficient use of light energy, that is efficient transport of light to back side edges of grooves or slits.

For the first structure, due to continuity of metal layer transport of energy is governed by phenomenon of tunneling of plasmons from front to back surface where they are scattered into free space and interfere constructively in farfield region on the axis. Due to relatively high absorption in metal transmission does not exceed 30\% of incident energy. 
For the second lens waveguiding mechanism of plasmonic modes for small slits, which propagation constant $\beta$ can be calculated from the equation

$$
\tanh \left(\sqrt{\beta^{2}-k_{0}^{2} \varepsilon_{d}} \frac{w}{2}\right)=\frac{-\varepsilon_{d} \sqrt{\beta^{2}-k_{0}^{2} \varepsilon_{m}}}{\varepsilon_{m} \sqrt{\beta^{2}-k_{0}^{2} \varepsilon_{d}}}
$$

where $\mathrm{k}_{0}$ is wave vector of incident beam in free space, $w$ is slit width, $\varepsilon_{\mathrm{d}}$ and $\varepsilon_{\mathrm{m}}$ are the relative dielectric constants for dielectric in slits and metal, respectively. Combined plasmonic and propagation modes for wider chanels allows for higher transmission over $50 \%$. Focus formation by both structures is determined by geometrical parameters such as lattice constant $\Lambda$ which determines position of radiating point sources from the edges of the slits or grooves and wavelength to lattice constant ratio $\lambda / \Lambda$ which determines phase matching of propagating waves on the optical axis. Calculations made for metals such as silver, gold, copper and aluminum [14] show that different materials influence only the transmission which corresponds directly to imaginary part of dielectric function of considered metal and decide on absorption in the material.

\subsection{Method of analysis}

Due to axial symmetry of considered system calculation are performed using finite-difference time-domain method (FDTD) in cylindrical coordinates with applied Body of Revolution (BoR) formalism, in which electric $\mathrm{E}(\mathrm{r}, \varphi, \mathrm{z}, \mathrm{t})$ and magnetic $\mathrm{H}(\mathrm{r}, \varphi, \mathrm{z}, \mathrm{t})$ fields in the azimuthal direction $\phi$ are expressed by analytical formulas

$$
\begin{gathered}
E(r, \phi, z, t)=\sum_{m=0}^{\infty} E_{c, m}(r, z, t) \cos m \phi+\sum_{m=0}^{\infty} E_{s, m}(r, z, t) \sin m \phi, \\
H(r, \phi, z, t)=\sum_{m=0}^{\infty} H_{c, m}(r, z, t) \cos m \phi+\sum_{m=0}^{\infty} H_{s, m}(r, z, t) \sin m \phi,
\end{gathered}
$$

where $m$ is the azimuthal mode number, and this analytical dependence is incorporated into the FDTD code. Such approach allows for simplifying the computational burden to a two dimensional simulation in the longitudinal and radial directions, speeding the time of computations and computer resources saving. For simulation we used free-package MEEP [19] and inhouse BOR-FDTD code [17]. Metal is modeled using Drude model dispersion

$$
\varepsilon(\omega)=\varepsilon_{\infty}-\frac{\omega_{p}^{2}}{[\omega(\omega+i \Gamma)]},
$$

where $\varepsilon_{\infty}=3.7, \omega_{\mathrm{p}}=13,673 \mathrm{THz}, \Gamma=27.35 \mathrm{THz}$. The parameters for silver are fitted to data from Johnson and Christy [20]. Transmission properties of both nanolenses were calculated for spectral range from 450 to $700 \mathrm{~nm}$ and values of index of refraction changing from 1 to 2

\subsection{Optical properties of nanolenses}

\subsubsection{Lens with grooves}

Figure 2 shows in color scale transmission T of free-standing grooved lens made of silver layer of thickness $d=100 \mathrm{~nm}$, groove width and depth equal $w=100 \mathrm{~nm}$ and $h=40 \mathrm{~nm}$, respectively. Calculations are performed in plane $1 \mu \mathrm{m}$ behind back surface for similar ranges of wavelengths and lattice constants changing from 450 to $700 \mathrm{~nm}$. For the considered spectral range focus formation is observed in the region below black line. In this region the largest both focal length and full width at half maximum (FWHM) of focal spot are achieved for shortest wavelength and are decreasing with increase of wavelength. The black line indicates the set of wavelengths and lattice constants for which maximum resolution equal 0.42 $\lambda$ of grooved lens is achieved. Although, the size of focal spot is sub-wavelength, the focus formation due to constructive interference occurs in far-field region thus resolution of that lens is still diffraction limited. With increase of period of the grating the region of focus formation and transmission band with highest values widens and for longest $\Lambda=700 \mathrm{~nm}$ focusing occurs in nearly full visible spectrum. 
Focusing region in $(\Lambda, \lambda)$-space does not depend on metal [14] and thus focusing phenomenon is determined by geometric parameters of the lens only. The largest impact on efficiency of the lens has wavelength-to-lattice constant $\lambda / \wedge$ ratio which influences the phase of spherical waves generated on back side grooves edges which interfere constructively on the optical axis in far-field region. Well shaped and symmetrical focal spot is formed due to constructive interference of longitudinal component $E_{z}$ and destructive interference of radial component $E_{r}$ of electric field emerged from back surface.

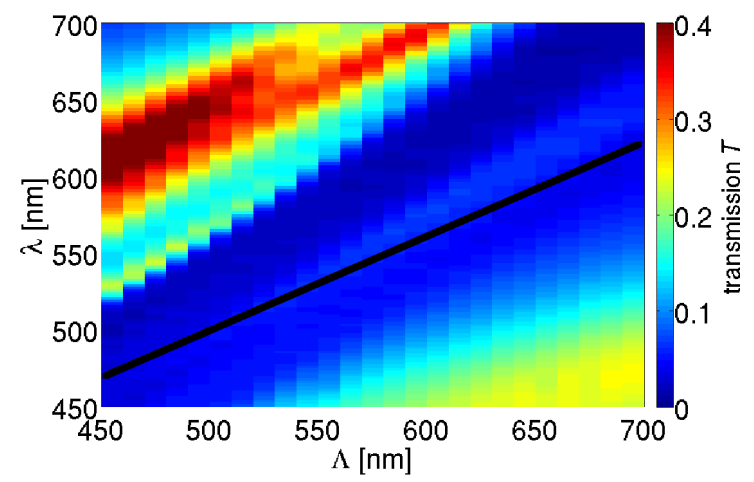

Fig. 2. Transmission $T$ through free-standing silver lens with grooves calculated for range of wavelengths and lattice constant $\Lambda$ from $450 \mathrm{~nm}$ to $700 \mathrm{~nm}$.

Free standing metal layer of thickness $100 \mathrm{~nm}$ is difficult to fabricate therefore it has to be integrated with a transparent medium. Figure 3 shows transmission through the above considered continuous, grooved metal film which is now embedded into dielectric substrate and thin protective layer on back surface. Calculations are performed for visible range wavelengths and dielectric constants changing from 1 to 2 . To illustrate influence of refractive index of dielectric matrix two lattice constants $\Lambda=600$ ad $700 \mathrm{~nm}$ are chosen from $(\Lambda, \lambda)$-space for which high transmission and focus formation in a broad spectrum occurs. For lattice constant $\Lambda=600 \mathrm{~nm}$ the maximum transmission of about $20 \%$ is a linear function of refractive index. It decreases from the maximum value of $20 \%$ with increase if $n$ values. The region of highest transmission becomes narrow and ends for wavelength-to-lattice ratio approaching unity, which corresponds to maximum resolution of the lens. Because focus formation is govern by geometric parameters as is explained above thus adding dielectric substrate does not alter width of focusing region. According to results showed in Figure 2 increase of a lattice constant leads to increase of transmission and broadening of focal region.
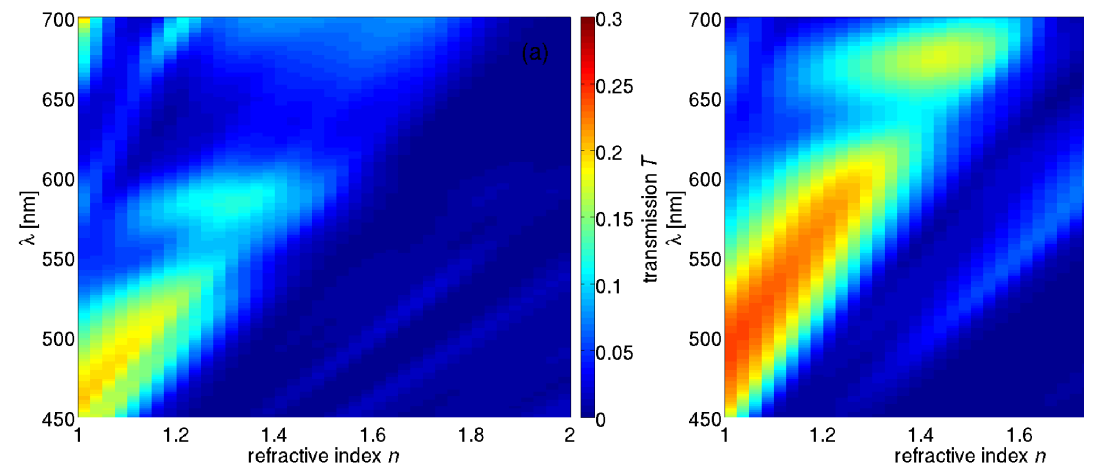

Fig. 3. Transmission through the silver grooved lens with width of grooves $w=100 \mathrm{~nm}$ as a function of refractive index $n$ and wavelength $\lambda$ calculated for lattice constant $\Lambda=600 \mathrm{~nm}$ (a) and $700 \mathrm{~nm}$ (b).

For lattice constant $\Lambda=700 \mathrm{~nm}$ region of maximum transmission of values close to $25 \%$ is shifted toward longer wavelength and ends for refractive index about 1.45. Thus application of dielectric substrate does not improve transmission properties of the grooved nanolens but allows for increase of transmission in the spectral region corresponding to the maximum lens resolution. This result leads to a conclusion that integration of the nanolens with dielectric substrate is possible without diminishing transmission properties for materials with refractive index smaller than value 1.45. Increase 
of lattice constant should improve level of transmission as well as width of region with focus formation but red-shift moves maximum resolution of the structure toward infrared range of light.

\subsubsection{Lens with slits}

Light transmission through the lens with slits is very different from tunneling through double sided grooves in the previous case. In the case of lenses with narrow slits light is guided as a plasmonic mode similarly as inside coaxial waveguides [14]. In the case of wide slits the predominant mechanism is a free space propagating mode not bound to the metal walls, however, plasmons at both inner and outer slit walls are still observed [14]. The wider are the slits, the more dominant the free space mode becomes, what leads to similarly high transmission through slit lenses made of different metals no matter how low or high losses they have.
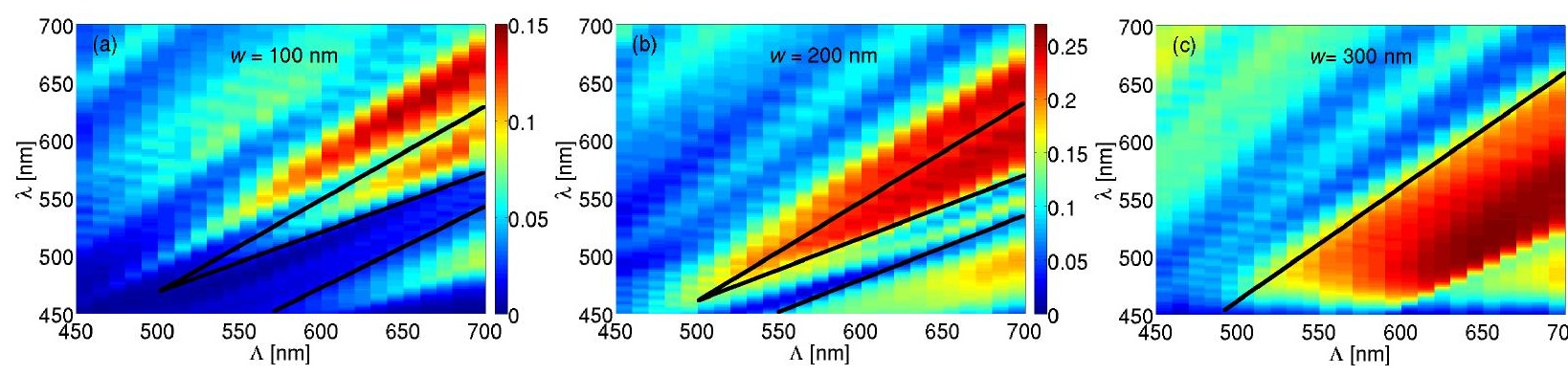

Fig. 4. Transmission through silver lens with slits integrated with dielectric matrix of refractive index $n=1.45$ as a function of wavelength $\lambda$ and lattice constant $\Lambda$ calculated for slit width (a) $100 \mathrm{~nm}$, (b) $200 \mathrm{~nm}$, (c) $300 \mathrm{~nm}$.

Figure 4 shows transmission through the second structure consisting of five concentric rings embedded in dielectric matrix with the same refractive index in core and cladding of a fiber which guides illuminating light, material that fills the slits and anticorrosion superstrate. For slits smaller than $300 \mathrm{~nm}$ focusing phenomenon occurs is observed in two separated transmission bands. In Figures $4 \mathrm{a}$ and $\mathrm{b}$ the short-wavelength and low-transmission bands appear bellow the lower black line. In Figures 4a and b high-transmission bands are contained in triangular regions between two upper black lines. Those bands widen for longer lattice constants. Similarly, as in the case of nanolens without on-axis hole, in those high-transmission bands FWHM decreases with increase of wavelength, however, resolution better than $0.5 \lambda$ is possible. For narrow slits waveguiding of plasmonic modes is responsible for transport of energy, small transmission for short wavelengths results from deeper penetration of electric field of SPPs into metal and thus strong absorption of energy. For nanolens with $300 \mathrm{~nm}$ slits (Fig. 4c) focus formation occurs in only one transmission band below the black line. Just below that line the maximum resolution equal $0.42 \lambda$ is achieved. For wide slits influence of directly propagating modes, which do not interact with slit walls, leads to broadening of high transmission band. For $300 \mathrm{~nm}$ wide slits transmission exceeds $40 \%$. Thus, for large lattice constants and wide slits our metallic nanolens assures large transmission within a wide range of wavelengths, covering almost all visible spectrum for which focusing occurs. Similarly, as in the case of nanolens without on-axis hole, focus formation by slit lens is govern by geometrical arrangement of the slits and the size of region of good focusing does not depend on type of metal [14].

To simulate influence of dielectric substrate on transmission and focusing properties of slit lens we choose two lattice constants $\Lambda=600$ and $700 \mathrm{~nm}$ for which high transmission and wide focusing region occurs. Figure 5 shows transmission through slit lens as a function of wavelength and refractive index of dielectric matrix. For the small lattice constant and slit width $w=100 \mathrm{~nm}$ a few bands of different transmission can be distinguished (Fig. 5a). A broad region with transmission close to $20 \%$ is observed for $n$ smaller than 1.4 . For wider slits the region with highest transmission broadens toward higher values of refractive index and reaches 1.8 for slits width equal $300 \mathrm{~nm}$. Focusing occurs for all considered $n$ in spectral region below $550 \mathrm{~nm}$. A change of dielectric substrate does not influence transmission which is almost uniformly high in interesting spectral range and its value depends on slit width only and reaches $45 \%$ for $w=300$ nm (Fig. 5c). For lattice constant $\Lambda=700 \mathrm{~nm}$ transmission band in $(\lambda, \mathrm{n})$-space slightly shifts toward small $n$ and considerable expands toward long wavelength. It results from phase-match condition which depends on the $\lambda / \Lambda$ ratio. In 
contrast to the lens with grooves the slit lens can be embedded in dielectrics with refractive indices from $1.3 \div 1.8$ range without decrease of transmission properties.
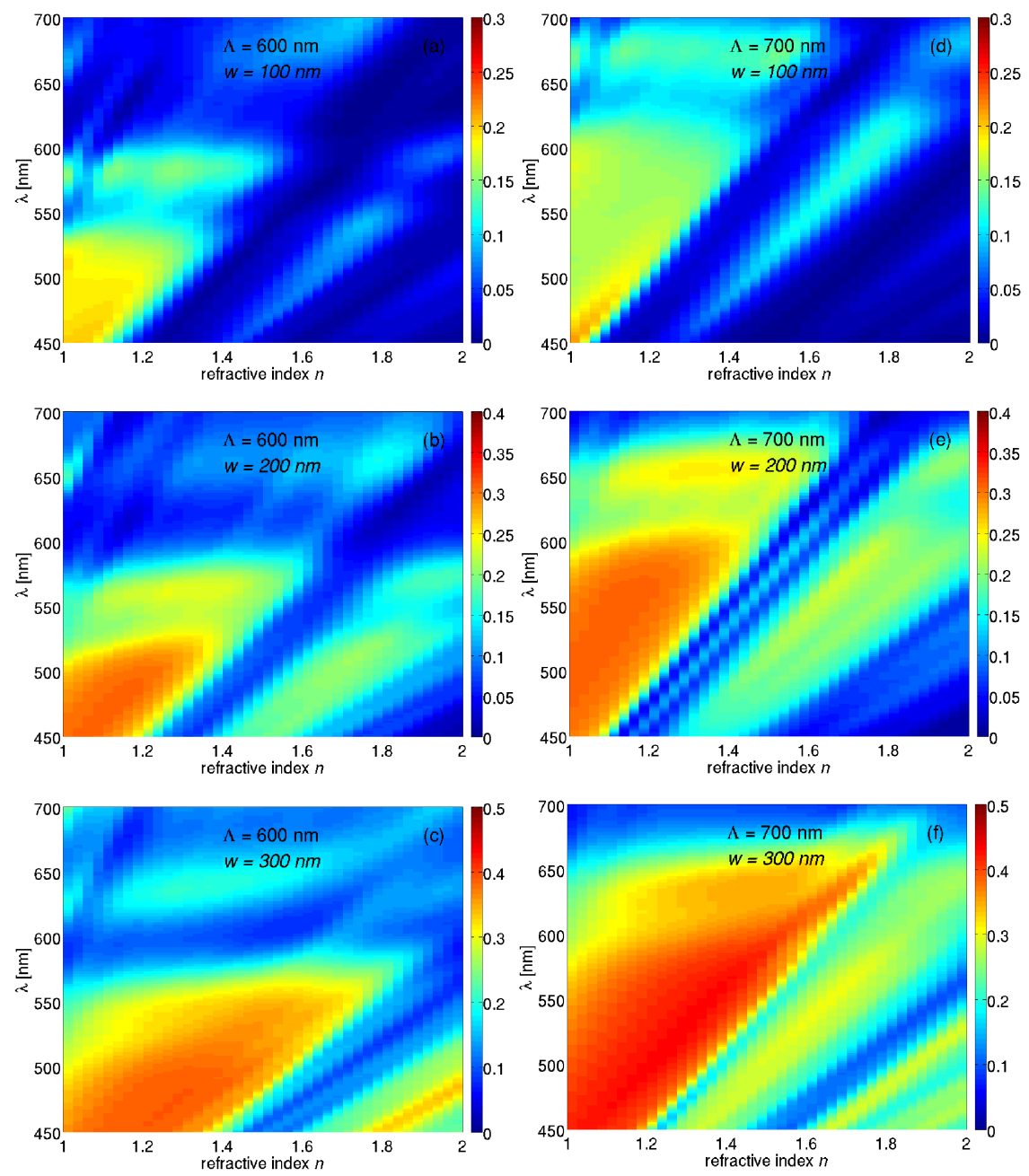

Fig. 5 Transmission through silver lens with slits as a function of refractive index $n$ and wavelength $\lambda$ calculated for slit width (a,d) $100 \mathrm{~nm}$, (b,e) $200 \mathrm{~nm},(\mathrm{c}, \mathrm{f}) 300 \mathrm{~nm}$ and lattice constant $\Lambda=600$ (a-c) and $700 \mathrm{~nm}$ (d-f).

\section{CONCLUSIONS}

In computer experiment we analyze two kinds of plasmonic lenses either grooved and continuous or perforated and integrated with a dielectric matrix. Focusing phenomenon of both lenses depends on geometric parameters (metal layer thickness $d$, slit and groove width $w$, lattice constant $\Lambda$, groove depth $h$ and on-axis stop diameter s). Sub-wavelength focusing is possible within a broad spectrum of wavelengths for the $\lambda / \Lambda$ ratio approaching unity. In continuous lens with double-sided grooves transmission does not exceed $30 \%$ for an optimized structure. In lens with wide slits waveguiding of plasmons and directly propagating modes leads to increase of transmission over $40 \%$. Integration of grooved lens with a dielectric matrix of increasing refractive index redshifts the high transmission region. For such materials as $\mathrm{MgF}, \mathrm{CaF}$ and other fluorides with $n<1.4$ transmission of grooved lens increases in spectral region with high resolution. Lens with slits can be integrated with substrates with $n$ reaching 1.8 without diminishing its focusing and transmission properties. Nanolenses of both types are resistant to temperatures up to 800 C. 


\section{ACKNOWLEDGEMENTS}

This work was supported by the Polish Ministry of Science and Higher Education under the project N N202 302638 and the National Centre for R\&D under the project N R15 0018 06. The authors are partners in COST Actions MP 0702 and MP 0803. Simulations were performed in the ICM, University of Warsaw, grant \#G33-7.

\section{REFERENCES}

[1] Gramotnev, D. K. and Bozhevolnyi, S. I., "Plasmonics beyond the diffraction limit,” Nature Photon. 4, 83-91 (2010).

[2] Yongqi Fu and Xiuli Zhou, “Plasmonic Lenses: A Review,” Plasmonics 5, 287-310 (2010).

[3] Sun, Z. and Kim, H. K., "Refractive transmission of light and beam shaping with metallic nano-optic lenses," Appl. Phys. Lett. 85, 642-644 (2004).

[4] Liu, Z., Steele, J. M., Srituravanich, W., Pikus, Y., Sun, C. and Zhang, X., "Focusing Surface Plasmons with a Plasmonic Lens,” Nano Lett. 5, 1726-1729 (2005).

[5] Srituravanich, W., Pan, L., Wang, Y., Sun, C., Bogy, D.B. and Zhang, X., "Flying plasmonic lens in the near field for high-speed nanolithography,” Nature Nanotechnology 3, 733-737 (2008).

[6] Lin, D. Z., Chen, C. H., Chang, C. K., Cheng, T. D., Yeh, C. S., and Lee, C. K., "Subwavelength nondiffraction beam generated by a plasmonic lens,” Appl. Phys. Lett. 92, 233106 (2008).

[7] Yuan, H. X., Xu, B. X., Lukiyanchuk, B. and Chong, T. C., "Principle and design approach of flat nano-metallic surface plasmonic lens,” Appl. Phys. A 89, 397-401 (2007)

[8] Shi, H., Wang, C., Du, C., Luo, X., Dong, X. and Gao, H., "Beam manipulating by metallic nano-slits with variant widths," Opt. Express 13, 6815-6820 (2005). http://www.opticsinfobase.org/oe/abstract.cfm?URI=oe-13-18-6815

[9] Kim, H. C., Ko, H., and Cheng, M., "High efficient optical focusing of a zone plate composed of metal/dielectric multilayer,” Opt. Express 17, 3078-3083 (2009). http://www.opticsinfobase.org/abstract.cfm?URI=0e-17-5-3078

[10] Chang, C. K., Lin, D. Z., Yeh, C. S., Lee, C. K., Chang, Y. C., Lin, M. W., Yeh, J. T., and Liu, J. M., "Experimental analysis of surface plasmon behavior in metallic circular slits,” Appl. Phys. Lett. 90, 061113 (2007).

[11] Mote, R. G., Yu, S. F., Ng, B. K., Zhou, W. and Lau, S. P., "Near-field focusing properties of zone plates in visible regime - New insights,” Opt. Express 16, 9554-9564 (2008).

[12] Wróbel, P., Pniewski, J., Antosiewicz, T. J. and Szoplik, T., "Focusing radially polarized light by concentrically corrugated silver film without a hole,” Phys. Rev. Lett. 102, 183902 (2009).

[13] Yu, Y. Y., Lin, D. Z., Huang, L. S., and Lee, C. K., "Effect of subwavelength annular aperture diameter on the nondiffracting region of generated Bessel beams,” Opt. Express 17, 2707-2713 (2009). http://www.opticsinfobase.org/oe/abstract.cfm?URI=0e-17-4-2707

[14] Wróbel, P., Antosiewicz, T. J. and Szoplik, T., "Plasmonic nanolenses made of noble metals focusing into farfield", Proc. SPIE 7746, 77461B (2010); doi:10.1117/12.883838

[15] Zhan, Q., “Cylindrical vector beams: from mathematical concepts to applications,” Adv. Opt. Photon. 1, 1-57 (2009). http://www.opticsinfobase.org/aop/abstract.cfm?URI=aop-1-1-1

[16] Antosiewicz, T. J. and Szoplik, T., "Description of near- and far-field light emitted from a metal-coated tapered fiber tip,” Opt. Express 15, 7845-7852 (2007) http://www.opticsinfobase.org/abstract.cfm?URI=0e-15-12-7845.

[17] Antosiewicz, T. J., Wróbel, P., and Szoplik, T., "Nanofocusing of radially polarized light with dielectric-metaldielectric probe,” Opt. Express 17, 9191-9196 (2009). http://www.opticsinfobase.org/oe/abstract.cfm?URI=oe-17$\underline{11-9191}$

[18] Chang, C. K., Lin, D. Z., Chang, Y. C., Lin, M. W., Yeh, J. T., Liu, J. M., Yeh, C. S. and Lee, C. K. “Enhancing intensity of emitted light from a ring by incorporating a circular groove,” Opt. Express 15, 15029-15034 (2007). http://www.opticsinfobase.org/abstract.cfm?URI=0e-15-23-15029

[19] Oskooi, A. F., Roundy, D., Ibanescu, M., Bermel, P., Joannopoulos, J. D. and Johnson, S. G., “MEEP: A flexible free-software package for electromagnetic simulations by the FDTD method," Computer Physics Communications 181, 687-702 (2010).

[20] Johnson P., Christy R., “Optical constants of the noble metals.” Phys Rev B 6, 4370-4379 (1972). 